Check for updates

Cite this: Nanoscale Adv., 2019, 1, 728

\title{
A random laser based on electrospun polymeric composite nanofibers with dual-size distribution
}

\author{
Mário César Albuquerque de Oliveira, ${ }^{a}$ Leonardo de Souza Menezes, (D) *b \\ Pablo I. R. Pincheira, ${ }^{c}$ Carlos Rojas-Ulloa, ${ }^{d}$ Nikifor Rakov Gomez, ${ }^{a}$ \\ Helinando Pequeno de Oliveira (D ${ }^{a}$ and Anderson Stevens Leônidas Gomes ${ }^{\text {ba }}$
}

\begin{abstract}
Electrospun fiber-based random lasers are environment-friendly flexible systems in which waveguiding/ scattering processes provided by their structure with a broad distribution of diameters are essential elements to generate a suitable lasing mechanism. In this work, we prepared electrospun fibers with dual-size diameter distribution (above and below the critical value for waveguiding), allowing that both optical processes can be established in the polymer network. As a result, random laser emission was observed for the electrospun fibers presenting dual-size diameters with rhodamine 6G as the gain medium, characterizing the combination of waveguiding/scattering as an adequate condition for development of organic nanofibrous random lasers. Degradation assays were also performed in order to evaluate the prolonged use of such random laser systems.
\end{abstract}

Received 9th October 2018

Accepted 3rd November 2018

DOI: $10.1039 / c 8 n a 00277 k$

rsc.li/nanoscale-advances

scattering along the fibers as well as multidirectional propagation, due to their disordered structure ${ }^{16}$ which depends on the fiber diameter scale. ${ }^{17}$ The importance of production of lightemitting electrospun nanofibers has been proved in the literature by the increasing, but still small, number of publications exploring random lasing by controlling the local disorder from doping with metal oxide nanoparticles such as $\mathrm{TiO}_{2}$ (ref. 18) or exploiting different organic dyes as the gain medium. ${ }^{19}$ These electrospun fibers can be a man-made engineered alternative to natural scattering materials such as egg shell membran $\mathrm{e}^{20}$ and flexible biocellulose. ${ }^{12}$

The morphology of the electrospun fibers is strongly affected by the experimental setup for the electrospinning procedure. Parameters such as polymer concentration, electric field strength (infusion rate of solution), viscosity, nature of solvent, surface tension, distance of the spinneret to the grounded target and net charge density ${ }^{21}$ are extremely relevant in order to produce regular and defect-free fibers. In terms of viscosity, high values are prohibitive because of the high solution cohesiveness, while low viscosity is conducive to the formation of irregular fibers and/or a high density of beads on fibers. Thus, the combination of polymers is a good strategy to reach adequate conditions for production of regular fibers, being an important tool that is available for use in the optimization of the fiber production process with improved chemical and physical properties.

The acetate ester of cellulose, cellulose acetate (CA), has been

${ }^{a}$ Graduate Program in Materials Science, Universidade Federal do Vale do São Francisco, Juazeiro, BA 48902-310, Brazil

${ }^{b}$ Departamento de Física, Universidade Federal de Pernambuco, Av. Prof. Moraes Rego, 1235, Cidade Universitária, Recife, PE 50670-901, Brazil. E-mail: lmenezes@df.ufpe.br

${ }^{c}$ Departamento de Ciencias Físicas, Universidad de La Frontera, Temuco, Chile

${ }^{d}$ Departamento de Ingeniería Mecánica, Universidad de La Frontera, Temuco, Chile obtained from the reaction of cellulose with acetic anhydride and sulfuric acid. ${ }^{22}$ Its use as a template for electrospun fiber production has attracted tremendous attention due to the 
unique niche in a plethora of environment-friendly applications of CA, such as development of antimicrobial membranes, ${ }^{23} \mathrm{drug}$ delivery systems ${ }^{22,24}$ and separators for lithium-ion batteries. ${ }^{25}$ The production of ultrathin electrospun fibers of CA has been reported in the literature as an important tool for different applications. $^{21}$

On the other hand, poly(ethylene oxide) (PEO) is considered as an important additive for incorporation into polymeric solutions for electrospinning. It has been reported that PEO incorporation into a conventional electrospun matrix reinforces the mechanical properties of resulting fiber ${ }^{26,27}$ and contributes to the synthesis of arrays with dual-size (diameter) distribution. ${ }^{22}$ As a consequence, new different properties and functionalities have been observed for PEO-modified electrospun fibers, such as microadhesive properties for drug delivery systems ${ }^{28}$ and ultra-toughened fibers of modified poly(methyl methacrylate)..$^{29}$

The production of ultrathin fibers of CA + PEO (tens of nanometers in diameter) could introduce important properties related to the dual distribution of diameters and mechanical reinforcement provided by PEO to CA electrospun fibers. The development of fibers with dual-size distribution applied as a support for the production of flexible RLs represents an important condition in which waveguiding and scattering can be combined in a mirror-free prototype for laser-like light emission. Gang Lv et al. ${ }^{17}$ reported that fiber-type cylindrical waveguides (PVA-based fibers) have limitations in presenting $\mathrm{RL}$ behavior for fiber diameters below $255 \mathrm{~nm}$. In their case, it was necessary to add titanium dioxide to the precursors (polymer melts) to increase their scattering properties, allowing RL emission in fibers with a diameter of the order of $150 \mathrm{~nm}$. These values were obtained from the assumption that propagation of light in fibers is established by a number $m$ of transverse magnetic (TM) modes given by eqn (1): ${ }^{18}$

$$
m<\left(\frac{2 d}{\lambda}\right) \sqrt{n_{\mathrm{nw}}^{2}-n_{0}^{2}}
$$

where $d$ is the diameter of the fibers, $\lambda$ is the wavelength of the guided light, and $n_{\mathrm{nw}}$ and $n_{0}$ are the refractive indices of polymer and air, respectively. Applying the refractive index of cellulose acetate $\left(1.54<n_{\mathrm{CA}}<1.62\right)^{30}$ and poly(ethylene oxide) $\left(n_{\mathrm{PEO}}=1.456\right.$ (ref. 31)) in eqn (1), it is possible to identify the limit (in terms of the nanofiber diameter) for light propagation in nanofibers in the range of diameters between $208 \mathrm{~nm}$ and $251 \mathrm{~nm}$. Based on a numeric analysis performed in ref. 17 it was observed that above this critical diameter, light is waveguided into fibers (due to the higher refractive index in comparison with air). At short distances, it tends to escape, favoring the scattering from thin fibers. It is therefore possible to improve the RL emission, providing a lower lasing threshold by fostering the incorporation of additional scatterers, i.e., the fibers with diameters of about tens of nanometers in addition to thicker fibers.

In this work, we have explored the incorporation of PEO into the CA template for electrospinning in order to evaluate the influence of dual-size distribution of electrospun fiber diameters on RL effects, due to the competition established by waveguiding and scattering in thick/thin nanofibers. Rhodamine 6G (Rh6G) was employed as the gain medium.

\section{Materials and methods}

\section{Materials}

Acetyl cellulose and poly(ethylene oxide) (Sigma Aldrich), dichloromethane (DCM) (Vetec), Rh6G (Sigma Aldrich), and methanol (MeOH) (Vetec) were used as received. Polymeric support solutions for electrospinning were prepared as follows: acetyl cellulose $(10 \mathrm{wt} \%)$ was dispersed in a DCM/MeOH mixture $(4: 1 \mathrm{v} / \mathrm{v})$ and kept under intense stirring for complete dissolution of the components. Rh6G (2 mM) was incorporated into the polymeric solution (additional stirring for $2 \mathrm{~min})$. Finally, PEO $(0,10$ and $20 \mathrm{wt} \%)$ is loaded into the mixture and dispersed under stirring for an additional $2 \mathrm{~min}$.

\section{Electrospinning procedure}

A freshly prepared solution was disposed into a $3 \mathrm{~mL}$ syringe to be applied in a typical electrospinning assay. For this, the syringe was connected to an infusion pump and kept at fixed pressure and infusion rate $\left(1 \mathrm{~mL} \mathrm{~h}^{-1}\right)$. A high voltage source $(12 \mathrm{kV})$ was connected to the spinneret of the needle that was disposed at $10 \mathrm{~cm}$ from a grounded target. The electrospun fibers were deposited on aluminum foil at humidity lower than $50 \%$. The incorporation of PEO at relative concentrations of $10 \%$ and $20 \%$ results in a well-defined dual distribution of diameters with a shift to thicker fibers as compared to the case of no incorporated PEO ( $0 \%$ PEO), for which a single peaked size (diameter) distribution is observed (see below).

\section{Random laser characterization}

The lasing emission spectra of three different samples were measured with a pulsed Nd:YAG laser (Brio - Quantel, $5 \mathrm{~Hz}$, $5 \mathrm{~ns}, \lambda=532 \mathrm{~nm}$ ), delivering a maximum energy of $20 \mathrm{~mJ}$. The excitation light was perpendicularly directed onto the sample, and gently focused onto a diameter of $\sim 3 \mathrm{~mm}$ at the sample surface, and the emitted radiation was collected in the reflection mode at $\sim 45^{\circ}$ by a multimode optical fiber. The RL emission was spectrally analysed using an Ocean Optics USB4000 spectrometer (spectral resolution $\sim 1.5 \mathrm{~nm}$ ). The results for all three samples were recorded under identical experimental conditions and all the measurements were carried out at room temperature. Fig. 1 shows the experimental setup (the inset shows photographs of the sample (CA $+10 \%$ PEO) under no excitation, excited below and above the random laser threshold).

\section{Results and discussion}

\section{Structural and morphological characterization}

The structure of the synthesized electrospun fibers was depicted by scanning electron microscopy (SEM) and further characterized by Raman spectra. SEM images in Fig. 2 reveal that electrospun fibers (pristine CA (CA + 0\% PEO) and CA + PEO) are free of defects, presenting a regular structure distributed on 

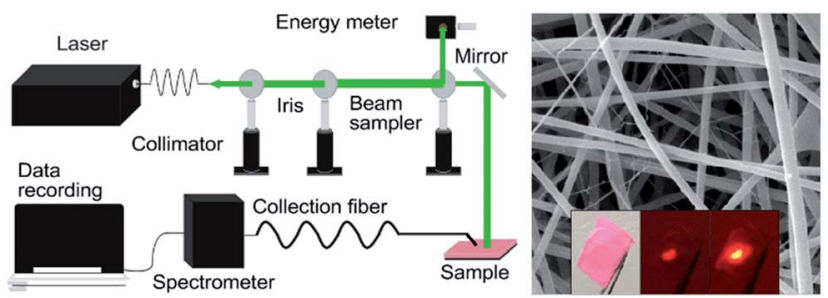

Fig. 1 Left: Experimental setup for RL emission detection. Right: SEM image of electrospun fibers (CA $+10 \%$ PEO) with photographs of the sample as produced: under room illumination (bottom left), and excited below (bottom middle) and above (bottom right) the random laser threshold.

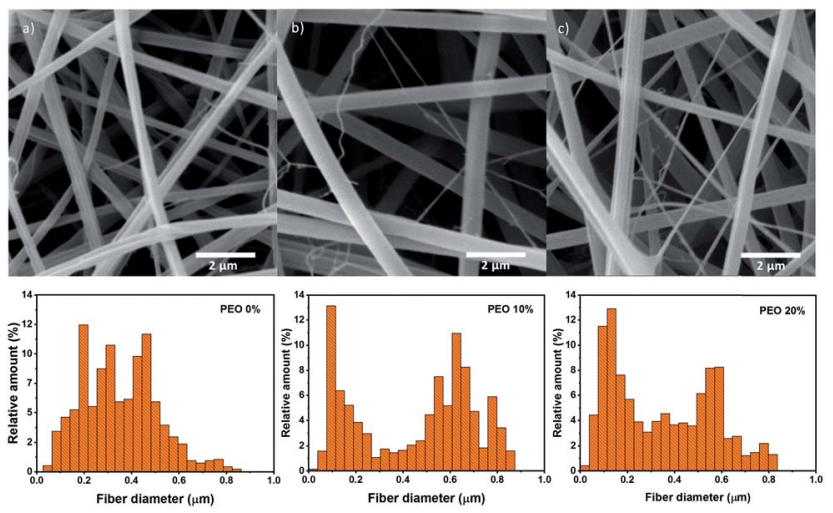

Fig. 2 SEM images of the electrospun fibers of (a) pristine cellulose acetate - CA + 0\% PEO (PEO-0\%), (b) cellulose acetate + 10 wt $\%$ PEO - CA + 10\% PEO (PEO-10\%), and (c) cellulose acetate + 20 wt $\%$ PEO CA $+20 \%$ PEO (PEO-20\%) and the corresponding diameter histograms of each sample.

a porous microscopic net with diameters in the range of $10 \mathrm{~nm}$ to $850 \mathrm{~nm}$, indicating that experimental setup conditions favor the split of the jet into smaller filaments that are connected in a fibrous membrane in the absence of beads. The distribution of diameters for $\mathrm{CA}+0 \%$ PEO electrospun fibers, indicated in Fig. 2(a), is characterized by a typical Gaussian distribution centered at $460 \mathrm{~nm}$ and a concentration of $18.78 \%$ of fibers with $d<200 \mathrm{~nm}$ and $81.22 \%$ of fibers with $d>200 \mathrm{~nm}$. The incorporation of $10 \mathrm{wt} \%$ of PEO into the sample (CA $+10 \%$ PEO) (see Fig. 2(b)) results in a typical dual-size diameter distribution of fibers with an increase in the diameter of thick fibers to values of the order of $630 \mathrm{~nm}$ while a second distribution is clearly attributed to fibers with a diameter around $100 \mathrm{~nm}$.

For this composition, $70.65 \%$ of fibers present a diameter above $200 \mathrm{~nm}$, indicating that the two populations (thicker and thin fibers) are clearly identified in the resulting sample. With further incorporation of PEO (sample CA $+20 \%$ PEO), the characteristic dual-size distribution of fibers with peaks centered at $570 \mathrm{~nm}$ (thicker fibers) and $130 \mathrm{~nm}$ (thinner fibers) is observed. It is worth mentioning that PEO incorporation results in two different mechanisms in the electrospun fibers: dual-size distribution and decreasing diameter of synthesized fibers with increasing concentration of PEO - the relative amount of diameter with $d<200 \mathrm{~nm}$ increases by $35.40 \%$, see
Fig. 2(c). This means that waveguiding and scattering are favored in a low concentration regime of the additive (due to the higher concentration of thicker fibers and appearance of thin fibers) while a higher concentration of PEO favors the scattering due to the decreasing diameter of both populations (thinner and thicker fibers).

The chemical structure of the produced fibers was characterized by Raman spectroscopy (see Fig. 3) in which the response of the samples CA $+0 \%$ PEO and mixed electrospun fibers CA $+10 \%$ PEO and CA $+20 \%$ PEO is investigated. Characteristic bands of CA are present at $1757 \mathrm{~cm}^{-1}(\mathrm{C}=\mathrm{O}$ bonds in the acetyl groups of the polymer chains), ${ }^{32} 1366 \mathrm{~cm}^{-1}$ (deformation vibrations of the CA backbone), ${ }^{32}$ 1106, 926, 823 and $640 \mathrm{~cm}^{-1}$ (assigned to, respectively, COC symmetric ring vibrations, $\mathrm{C}-\mathrm{H}$ bonds, stretching vibrations of $\mathrm{C}-\mathrm{O}$ groups and $\mathrm{C}-\mathrm{OH}$ bonds). ${ }^{32,33}$ The spectrum of CA + PEO fibers confirms the presence of characteristic bands of CA in addition to characteristic bands of PEO, observed at 1499, 1226, 1130 and $1044 \mathrm{~cm}^{-1}$ assigned to in-phase bending for the $\mathrm{CH}_{2}$ group, ${ }^{34}$ out-of-phase twisting of the $\mathrm{C}-\mathrm{H}$ group, ${ }^{35}$ and the stretching mode of the $\mathrm{C}-\mathrm{C}$ and $\mathrm{C}-\mathrm{O}$ groups, ${ }^{35,36}$ respectively.

These results confirm that PEO is successfully incorporated into the electrospun fibers, with a strong effect on the morphology of the resulting fibers.

\section{Random laser behavior}

The spectral narrowing behaviour of the studied samples and of a control sample composed of nanofibers made only with PEO as a function of the excitation energy is summarized in Fig. 4. In Fig. 4(a) and (b), the RL emission spectra of the sample CA + $10 \%$ PEO are shown for different excitation energies, from below to well above the laser threshold. As previously reported in ref. 13, which also employed Rh6G as the gain medium, the increasing pumping energy induces re-absorption and reemission of fluorescence, resulting in a bathochromic shift, as clearly seen in Fig. 4(b). The plot of the full width at half maximum (FWHM) of the emission spectra as a function of the excitation energy is shown in Fig. 4(c) for the three studied

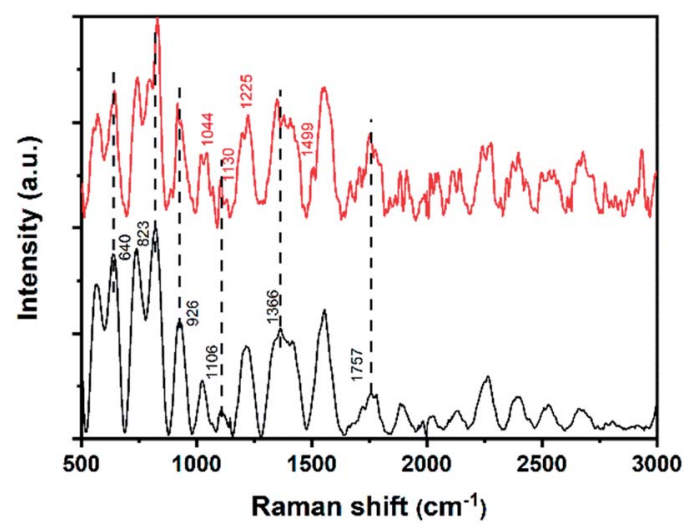

Fig. 3 Raman spectra of the electrospun fibers of CA + 0\% PEO (black curve) and electrospun fibers of CA $+10 \%$ PEO (red curve; vertically displaced for ease of visualization). 

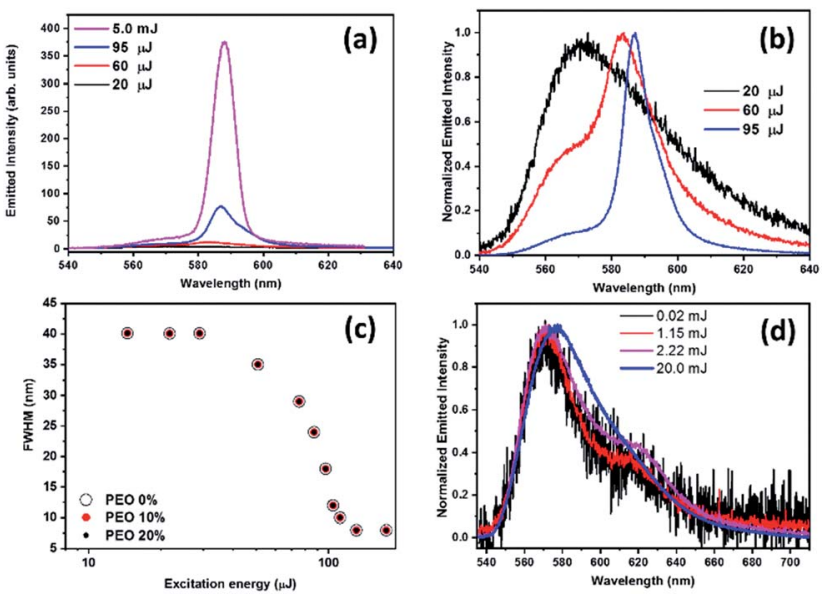

Fig. 4 (a) RL spectral emission versus excitation energy for the electrospun fibers of cellulose acetate $+10 w t \%$ PEO (CA + 10\%PEO) from below to well above the threshold. (b) Normalized emission spectra as in (a), clearly showing the bathochromic behavior from below to above the threshold. (c) Full width at half maximum (in $\mathrm{nm}$ ) as a function of the excitation energy $(\mu \mathrm{J})$ for the three studied samples CA $+0 \%$ PEO (PEO-0\%), CA + 10\% PEO (PEO-10\%) and CA + 20\% PEO (PEO-20\%). (d) Control experiment results on normalized fluorescent emission from $\mathrm{PEO}-100 \%$ (no CA) nanofibers, where no RL emission is observed (no linewidth narrowing), for powers from below to above the threshold.

samples. As can be seen, the linewidth is strongly reduced from $40 \mathrm{~nm}$ to $8 \mathrm{~nm}$ from below to well above the excitation threshold. A RL threshold of $97 \mu \mathrm{J}$ is inferred from Fig. 5 and is close to the value where the minimum linewidth is achieved.

In contrast, fibers of PEO only (PEO-100\%) prepared in the absence of CA showed negligible spectral narrowing at energy as high as $20 \mathrm{~mJ}$, which is over $200 \times$ higher than the threshold for the RL emission in the dual mode fibers with CA + PEO - see Fig. 4(d). In addition, no spectral shift was observed with increasing excitation energy density, as no laser emission was taking place. This is an indication that CA plays a critical role in lasing effects of the resulting fibers. PEO introduces an

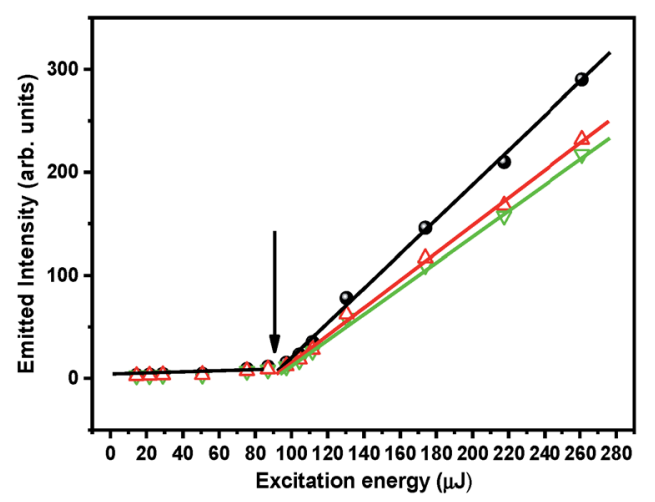

Fig. 5 RL emission intensity as a function of excitation energy for the samples studied: CA + 0\% PEO (red up triangles), CA + 10\% PEO (black circles) and CA $+20 \%$ PEO (down green triangles). The energy threshold is marked by the downward arrow, measured as $97 \mu \mathrm{J}$. The experimental error in determining the excitation energies is $\pm 10 \%$. additional feature into the RL system, with scattering from thinner fibers and improvement of mechanical properties, as the main additive. As a consequence, the emitted intensity is strongly affected by incorporation of the additive. The most efficient RL emission was observed for the sample CA $+10 \%$ PEO followed by CA $+20 \%$ PEO and CA $+0 \%$ PEO, as seen in Fig. 5.

Another relevant aspect to be reported refers to a red shift in the RL emission observed for samples prepared by adding PEO. This process is independent of the relative concentration of PEO employed in this study and can be associated with a dual distribution of fiber diameters that differs from a single distribution of diameters for CA $+0 \%$ PEO electrospun fibers. This result is shown in Fig. 6, for RL emission well above the threshold (pump energy of $5 \mathrm{~mJ}$ ). As discussed in the next section, the main role presented by the thinner nanofibers is to scatter the evanescent field of the light amplified in the thicker nanofibers, which increases the path of the emitted photons inside the RL medium, thus increasing reabsorption events, which in turn lead to a redshift of the resulting emitted light. A red-shift of $8.0 \mathrm{~nm}$ was measured for samples prepared with the incorporation of PEO.

\section{The location of the Rh6G molecules in the nanofiber matrix and the corresponding effects in the random lasing action}

In order to study how the active material is distributed in space (in and/or on/between the nanofibers), and to gain insight on how the optical gain works for the investigated RL system, we performed two assays on the homogeneity of the Rh6G molecule distribution in the nanofibers. These assays, which are helpful to evaluate a general scheme for processes involved in the RL emission in electrospun fibers, refer to different strategies to include the gain medium in the nanofiber matrix. In the first assay, the nanofibers were prepared by the conventional method - dispersion of the dye solution in the polymeric mixture before performing electrospinning at a high electric field. In the second scheme of preparation, the dye solution was not added to the polymeric material. From this pure polymeric mixture, without traces of Rh6G, the nanofibers were

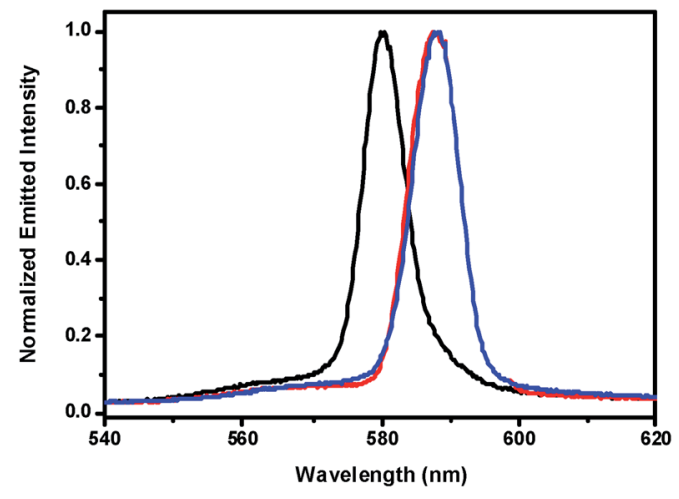

Fig. 6 Normalized random laser emission intensity of electrospun fibers as a function of incorporation of the additive PEO $10 \%$ - black line, $10 \%$ - red line and $20 \%$ - blue line) in the polymeric matrix of cellulose acetate. The pump energy was $5 \mathrm{~mJ}$. 
electrospun. Then, the Rh6G solution was dropcast on the electrospun nanofibers, so that the dye molecules were adsorbed on the nanofiber walls. These assays are schematized in Fig. 7. The RL emission spectra shown in the inset of Fig. 7(a) confirm that placing Rh6G molecules mainly onto the fibers favors a low threshold for RL emission.

On the other hand, when placing Rh6G on the surface of the nanofibers (Fig. 7(b)), the system exhibits negligible RL action at the same excitation energy (inset of Fig. 7(a)). However, by increasing the excitation energy, it is observed in Fig. 7(c) and in its inset that the RL effect is due to the possible process of scattering of light between adjacent fibers covered by the organic dye.

Here it is suitable to comment back on another result, shown in Fig. 5: the fact that the RL efficiency for the sample CA $+20 \%$ PEO is smaller than that for the sample CA $+10 \%$ PEO. One should consider that increasing the PEO doping level leads to an increase of the thinner nanofiber population, thus decreasing the population of thicker nanofibers. They, in turn, present more effective waveguiding properties and a larger gain, as shown in the inset of Fig. 7(a). Thus, increasing the PEO content of the nanofibers reduces the effective gain of the system. Therefore, it is reasonable to conclude that the main role played by the dye-filled thicker nanofibers is to provide gain

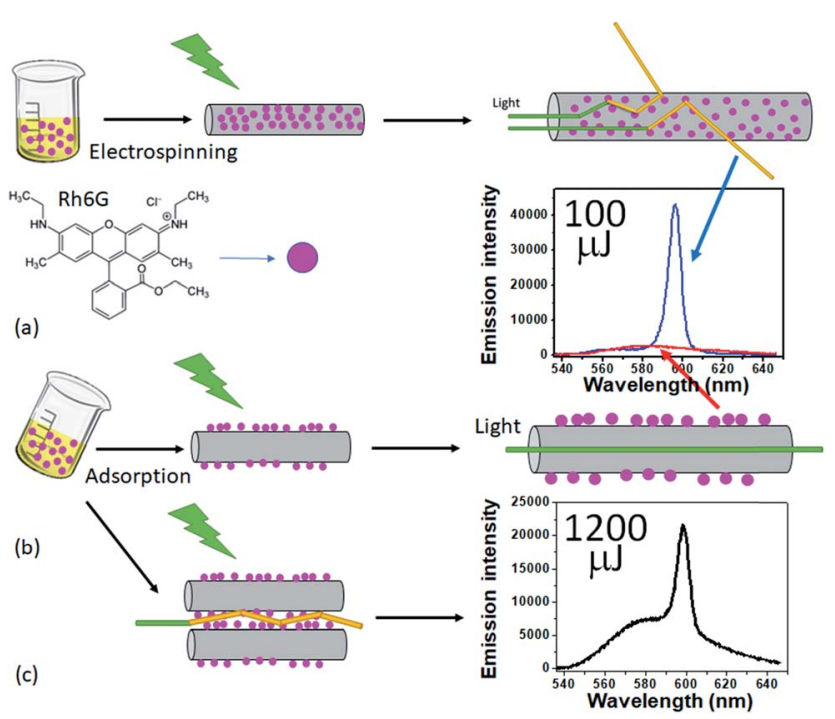

Fig. 7 Schematics of incorporating Rh6G, the gain medium of the studied RL, into the nanofiber matrix. (a) Conventional strategy: Rh6G solution is added to the polymeric mixture before performing electrospinning, leading to the molecules to be mainly inside the resulting nanofibers. The inset shows the RL emission spectrum for a pump energy of $100 \mu \mathrm{J}$ (blue line). (b) Drop-casting the Rh6G solution on the previously electrospun nanofibers, which causes the Rh6G molecules to be mainly adsorbed on the already existing nanofibers. The resulting emission spectrum for a pump energy of $100 \mu \mathrm{J}$ can also be seen in the inset of (a) (red line), which shows no RL action. (c) Sketch of the RL feedback mechanism in the case of the Rh6G molecules adsorbed on the surface of the nanofibers. The inset shows the resulting emission when the system is excited with $1200 \mu \mathrm{J}$ (more than $12 \times$ larger than the pump energy threshold), still showing some fluorescent background together with a $\mathrm{RL}$ emission peak. The results are for the sample CA $+10 \%$ PEO.

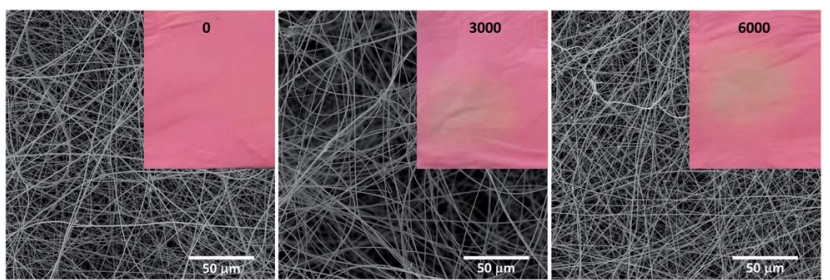

Fig. 8 Influence of long-term high intensity light incidence on the degradation of CA $+20 \%$ PEO electrospun fibers as a function of the number of shots (excitation energy: $5 \mathrm{~mJ}$ ). Insets: photographs of the sample, after being excited by a number of excitation laser shots indicated by the numbers.

in the system, while the thinner nanofibers contribute more effectively to the scattering of light in the evanescent field of the guided modes in the thicker nanofibers.

\section{Random laser degradation studies}

An important issue in RL using dyes and a host scattering medium is the RL degradation, which normally is not reported. It was observed from SEM images, shown in Fig. 8, that negligible degradation is observed in the electrospun fibers after 6000 shots of the pump laser with $5 \mathrm{~mJ}$ (well above the threshold), characterizing an important result for prolonged use of the organic matrix as a component for RL emission. On the other hand, as can be seen from the photographs of the samples, after 6000 shots one observes bleaching of the dye molecules in the matrix.

The long-term degradation of the random laser was also characterized by the measurement of the emitted intensity and full width at half maximum (FWHM) as a function of the number of shots, as shown in Fig. 9. As can be seen, and as expected from what is shown in Fig. 8, the RL intensity falls down to $74 \%$ after 2200 shots at $1 \mathrm{~Hz}$, which implies a working time of $\sim 37$ minutes. It should be noticed that the FWHM broadens by $66 \%$ from the initial narrow random laser emission. This behavior is known for several nanocomposite based random lasers where the gain medium is an organic dye. It can

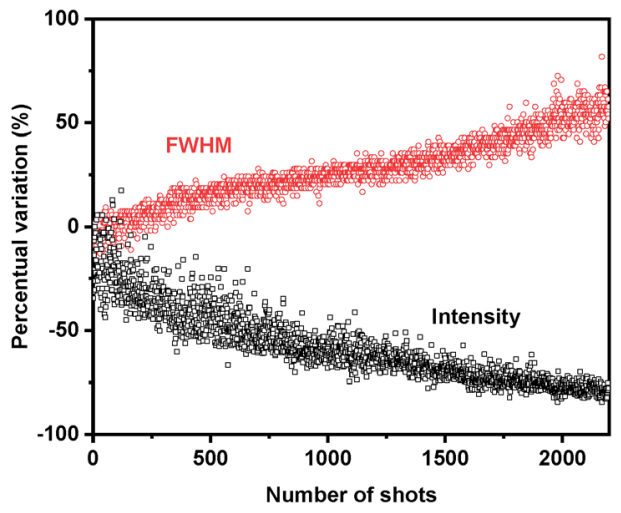

Fig. 9 Degradation behavior of the FWHM and intensity of RL as a function of the number of shots. The excitation energy was $5 \mathrm{~mJ}$ and the repetition rate was $1 \mathrm{~Hz}$. 
be overcome by appropriate scattering nanomaterial treatment, as demonstrated in ref. 37, where $\mathrm{TiO}_{2}$ nanoparticles were specially treated to extend their working lifetime for RL emission in contact with the dye. Alternatively, different scatterers can be used, as demonstrated with $\mathrm{SiO}_{2}$ (ref. 38) which also minimizes the degradation of the dye + nanoparticle RL.

In the present case, since there is no damage to the nanofibers, other gain media can be used, such as rare earth nanoparticles or $\mathrm{ZnO}$, therefore avoiding the chemically induced degradation process which occurs with dyes. In particular, $\mathrm{ZnO}$ has already been prepared with nanofibers for other purposes (such as photocatalysts ${ }^{39}$ ) which implies that it is feasible. $\mathrm{ZnO}$ RL has also been reported in several occasions. ${ }^{40}$

\section{Conclusion}

The development of environment-friendly electrospun fibers with a dual-size distribution of diameters represents a promising procedure for production of organic random lasers based on an adequate combination of waveguiding and scattering mechanisms. Such a combination of electrospun arrays of thin and thick fibers also characterizes an adequate support in which mechanically reinforced fibers (by PEO incorporation) improve the prolonged action of electrospun fibers as random lasers. The dye based RL degradation observed for our samples can be overcome by changing the gain medium, and work is underway using $\mathrm{ZnO}$ decorated dual-size electrospun nanofibers.

\section{Conflicts of interest}

There are no conflicts to declare.

\section{Acknowledgements}

This work was partially supported by the Brazilian agencies FINEP, CAPES, FAPESB, FACEPE, CNPq and Instituto Nacional de Fotônica - INFo. Pablo I. R. Pincheira acknowledges support from FONDECYT No. 3180696.

\section{References}

1 M. Y. Lee, J. Hong, E. K. Lee, H. Yu, H. Kim, J. U. Lee, W. Lee and J. H. Oh, Adv. Funct. Mater., 2016, 26, 1445-1453.

2 S. S. Gandhi, Y. Li, D. Luo and L. C. Chien, J. Polym. Sci., Part B: Polym. Phys., 2018, 56, 551-557.

3 F. Luan, B. Gu, A. S. L. Gomes, K.-T. Yong, S. Wen and P. N. Prasad, Nano Today, 2015, 10, 168-192.

4 N. M. Lawandy, R. Balachandran, A. S. L. Gomes and E. Sauvain, Nature, 1994, 368, 436.

5 L. Sznitko, J. Mysliwiec and A. Miniewicz, J. Polym. Sci., Part B: Polym. Phys., 2015, 53, 951-974.

6 Y.-J. Lee, C.-Y. Chou, Z.-P. Yang, T. B. H. Nguyen, Y.-C. Yao, T.-W. Yeh, M.-T. Tsai and H.-C. Kuo, Nanoscale, 2018, 10, 10403-10411.

7 M. V. Santos, É. Pecoraro, S. H. Santagneli, A. L. Moura, M. Cavicchioli, V. Jerez, L. A. Rocha, L. F. C. de Oliveira,
A. S. L. Gomes and C. B. de Araújo, J. Mater. Chem. C, 2018, 6, 2712-2723.

8 T. Zhai, J. Chen, L. Chen, J. Wang, L. Wang, D. Liu, S. Li, H. Liu and X. Zhang, Nanoscale, 2015, 7, 2235-2240.

9 S. Kim, S. Yang, S. H. Choi, Y. L. Kim, W. Ryu and C. Joo, Sci. Rep., 2017, 7, 4506.

10 R. R. da Silva, C. T. Dominguez, M. V. dos Santos, R. BarbosaSilva, M. Cavicchioli, L. M. Christovan, L. S. de Melo, A. S. L. Gomes, C. B. de Araújo and S. J. Ribeiro, J. Mater. Chem. C, 2013, 1, 7181-7190.

11 S. P. Lau, H. Yang, S. F. Yu, C. Yuen, E. S. Leong, H. Li and H. H. Hng, Small, 2005, 1, 956-959.

12 M. V. dos Santos, C. T. Dominguez, J. V. Schiavon, H. S. Barud, L. S. de Melo, S. J. Ribeiro, A. S. L. Gomes and C. B. de Araújo, J. Appl. Phys., 2014, 115, 083108.

13 N. Ghofraniha, L. La Volpe, D. Van Opdenbosch, C. Zollfrank and C. Conti, Adv. Opt. Mater., 2016, 4, 1998-2003.

14 I. Viola, N. Ghofraniha, A. Zacheo, V. Arima, C. Conti and G. Gigli, J. Mater. Chem. C, 2013, 1, 8128-8133.

15 A. Camposeo, F. Di Benedetto, R. Stabile, A. A. Neves, R. Cingolani and D. Pisignano, Small, 2009, 5, 562566.

16 M. Montinaro, V. Resta, A. Camposeo, M. Moffa, G. Morello, L. Persano, K. Kazlauskas, S. Jursenas, A. Tomkeviciene and J. V. Grazulevicius, ACS Photonics, 2017, 5, 1026-1033.

17 G. Lv, D. Huang, S. Wu, G. Ren, W. Yang and T. Li, Laser Phys., 2018, 28, 075803.

18 D. Huang, T. Li, S. Liu, T. Yi, C. Wang, J. Li, X. Liu and M. Xu, Laser Phys., 2017, 27, 035802.

19 V. Resta, A. Camposeo, M. Montinaro, M. Moffa, K. Kazlauskas, S. Jursenas, A. Tomkeviciene, J. V. Grazulevicius and D. Pisignano, Opt. Express, 2017, 25, 24604-24614.

20 X. Liu, T. Li, T. Yi, C. Wang, J. Li, M. Xu, D. Huang, S. Liu, S. Jiang and Y. Ding, J. Mod. Opt., 2016, 63, 12481253.

21 H. Liu and Y. L. Hsieh, J. Polym. Sci., Part B: Polym. Phys., 2002, 40, 2119-2129.

22 O. Suwantong, P. Opanasopit, U. Ruktanonchai and P. Supaphol, Polymer, 2007, 48, 7546-7557.

23 R. Konwarh, N. Karak and M. Misra, Biotechnol. Adv., 2013, 31, 421-437.

24 S. Hu, Z. Qin, M. Cheng, Y. Chen, J. Liu and Y. Zhang, Cellulose, 2018, 25, 1883-1898.

25 D. Boriboon, T. Vongsetskul, P. Limthongkul, W. Kobsiriphat and P. Tammawat, Carbohydr. Polym., 2018, 189, 145-151.

26 C. Zhou, R. Chu, R. Wu and Q. Wu, Biomacromolecules, 2011, 12, 2617-2625.

27 F. Xiang, S. M. Ward, T. M. Givens and J. C. Grunlan, Soft Matter, 2015, 11, 1001-1007.

28 D. Jia, Y. Gao and G. R. Williams, Int. J. Pharm., 2017, 523, 376-385.

29 R. L. Andersson, V. Ström, U. W. Gedde, P. E. Mallon, M. S. Hedenqvist and R. T. Olsson, Sci. Rep., 2014, 4, 6335. 30 C. Tang and H. Liu, Composites, Part A, 2008, 39, 1638-1643. 31 S. Jeon, J. Lee, J. Andrade and P. De Gennes, J. Colloid Interface Sci., 1991, 142, 149-158. 
32 I. L. Liakos, A. M. Holban, R. Carzino, S. Lauciello and A. M. Grumezescu, Nanomaterials, 2017, 7, 84.

33 J. Sánchez-Márquez, R. Fuentes-Ramírez, I. Cano-Rodríguez, Z. Gamiño-Arroyo, E. Rubio-Rosas, J. Kenny and N. Rescignano, Int. J. Polym. Sci., 2015, 2015, 320631.

34 H. M. Alhusiki-Alghamdi and N. S. Alghunaim, J. Mod. Phys., 2015, 6, 414.

35 I. Elashmawi and L. Gaabour, Results Phys., 2015, 5, 105-110.

36 T. Yoshihara, H. Tadokoro and S. Murahashi, J. Chem. Phys., 1964, 41, 2902-2911.
37 P. I. Pincheira, A. F. Silva, S. I. Fewo, S. J. Carreño, A. L. Moura, E. P. Raposo, A. S. L. Gomes and C. B. de Araújo, Opt. Lett., 2016, 41, 3459-3462.

38 A. Brito-Silva, A. Galembeck, A. S. L. Gomes, A. J. Jesus-Silva and C. B. de Araújo, J. Appl. Phys., 2010, 108, 033508.

39 E. S. Araujo, B. P. da Costa, R. A. P. Oliveira, J. Libardi, P. M. Faia and H. P. de Oliveira, J. Environ. Chem. Eng., 2016, 4, 2820-2829.

40 C. T. Dominguez, M. d. A. Gomes, Z. S. Macedo, C. B. de Araújo and A. S. L. Gomes, Nanoscale, 2015, 7, 317-323. 